\title{
Outcomes of patients with advanced stage ovarian cancer with intestinal metastasis
}

\author{
Eyüp Kebapcı ${ }^{1}$, Varol Gülseren ${ }^{2}$, Cem Tuğmen ${ }^{1}$, Mehmet Gökçü르, Ulaş Solmaz ${ }^{2}$, İsmail Sert ${ }^{1}$, \\ Mustafa Kocaer², Mehmet Özer², Mustafa Ölmez ${ }^{1}$, Muzaffer Sancı² \\ ${ }^{1}$ Department of General Surgery, Tepecik Education and Research Hospital, Izmir, Turkey \\ ${ }^{2}$ Department of Gynecologic Oncology, Tepecik Education and Research Hospital, Izmir, Turkey
}

\begin{abstract}
Objectives: The aim of this study is to evaluate the results of advanced stage (stage IIIB-IVB) ovarian cancer (OC) patients with intestinal metastasis, and to investigate the factors that affect survival.

Material and methods: Patients who underwent cytoreductive surgery (CS) for FIGO stage IIIB-IVB OC with metastasis in the intestinal system, at Tepecik Research and Treatment Hospital between 2008-2014, were analyzed retrospectively. Patients with borderline ovarian tumor; those who had previously undergone radiation therapy and/or hysterectomy and patients having secondary or tertiary cytoreduction were excluded and 49 patients were included and analyzed in this study. Hysterectomy, bilateral salpingo-oopherectomy, pelvic and para-aortic lymph node sampling, resection of bulky lymph nodes and omentectomy were performed. Optimal cytoreduction was accepted as that which left residual tumor $\leq 1 \mathrm{~cm}$ maximum size.

Results: The risk factors affecting OS interval were investigated according to Cox' regression analysis. Optimality of the primary CS ( $p=0.008$ and HR $=5.202)$ and cancer stage $(p=0.016$ and HR $=6.083)$ were found to be statistically significant factors.

Conclusions: Achieving optimal CS is the most important aim for the general surgeon carrying out an intestinal resection procedure. Although resection procedures are superior in providing the desired optimal results when compared to excision surgery, their higher complication rates and subsequent lower quality of life must be taken into consideration when choosing either resection or excision methods; surgical intervention should always be kept to the minimum possible.
\end{abstract}

Key words: ovarian neoplasms, intestinal neoplasms, gynecologic surgical procedures

Ginekologia Polska 2017; 88, 10: 537-542

\section{INTRODUCTION}

Ovarian cancer (OC), being responsible for $40 \%$ of all gynecologic cancers in women, is the most lethal gynecological malignity [1]. OC generally expands through the abdominal cavity before becoming symptomatic and is most often diagnosed when local metastatic disease has already taken hold (International Federation of Gynecology and Obstetrics [FIGO] stage III and IV) [2, 3].

The most important prognostic factors in advanced stage $O C$ are the stage of the disease and the ability to perform optimal cytoreductive surgery (CS) during primary surgery $[4,5]$. Indeed, in advanced stage OC, the standard treatment with the best oncologic outcomes is primary CS followed by adjuvant chemotherapy (CT) $[3,6]$. The objective of primary debulking surgery is to achieve maximum cytoreduction [7]. CS comprises hysterectomy, bilateral salpingo-oopherectomy, omentectomy and complete resection of macroscopic peritoneal implants [5]. Because of its anatomic proximity to the woman's pelvic organs and the tendency of $O C$ to spread, rectosigmoid resection is the most commonly performed intestinal surgery procedure in a successful optimal cytoreduction [5]. If the tumor has infiltrated the pelvic sidewalls, en bloc resection of the tumor, uterus, adnexa and rectosigmoid is performed [5].

CS appears to be associated with a higher rate of intraoperative and early postoperative complications, such as 
bleeding, anastomotic leak and infection. In selected cases, ostomy is needed, which inevitably leads to a remarkable deterioration in the quality of the patient's life. At this point, it becomes debatable whether the advantages of optimal cytoreduction during primary treatment are sufficient to compensate for the risks brought by this extensive surgery.

The aim of this study is to evaluate the results of advanced stage (stage IIIB-IVB) OC patients with intestinal metastasis; and to investigate the factors that affect survival.

\section{MATERIAL AND METHODS}

Patients who underwent CS for FIGO stage IIIB-IVB OC with metastasis in the intestinal system, at Tepecik Research and Treatment Hospital between 2008-2014, were analyzed retrospectively. Patients with borderline ovarian tumor, a prior history of radiation therapy, prior hysterectomy and secondary or tertiary cytoreduction were excluded from the study. So totally 49 patients were included and analyzed in this study. The study group's surgical and pathology reports were evaluated in terms of disease-related features, surgical and postoperative factors, and long-term results. The study was approved by the local ethics committee at our institution.

The patients received a liquid diet and a mechanical bowel preparation was used one day prior to surgery. All surgical operations were carried out by experienced surgeons in the fields of general and gynecological oncologic surgery. A vertical midline incision was preferred in all patients for ease of access during exploration of the abdomen and organ resection. After entering the peritoneal cavity, ascites fluid was sampled if present; if not, peritoneal washing cytology was taken. During exploration of the abdominal cavity, peritoneal surfaces, omentum, colon and small intestine, paracolic, pelvic, mesenteric and para-aortic sites were all examined systematically and palpated to find any suspicious lesions. Hysterectomy, bilateral salpingo-oopherectomy; pelvic and para-aortic lymph node sampling; resection of bulky lymph nodes and omentectomy were all performed. Optimal cytoreduction was considered to be a residual tumor size $\leq$ one $\mathrm{cm}$ maximum, in accordance with the definition given by the Gynecologic Oncology Group 2006.

Our female patients came for follow-up evaluations every three months for the first two years, every six months for the next three years and annually thereafter. Computed tomography or magnetic resonance imaging was performed annually. The only criterion for ending follow-up was the death of the patient.

Survival analysis was based on the Kaplan-Meier method, and the results were compared using a log-rank test. Disease-free survival (DFS) was measured as the time between the primary surgery and detection of the first recurrence or the latest observation. Overall survival (OS) was the time between initial treatment and death or the latest observation. For statistical analysis, we used the $x^{2}$ test and Student's t-test for unpaired data. Cox' regression analysis was used to determine factors affecting survival, presented as hazard ratios (HR) and 95\% confidence intervals $(\mathrm{Cl})$. Data recording and statistical analyses were performed using SPSS (statistical package for the social sciences) software (version 17, SPSS, Inc, Chicago, IL). A p $<0.05$ was considered to indicate statistical significance.

\section{RESULTS}

Clinical features of the patients are shown in Table 1. The mean patient age was $54.1 \pm 10.6$ and mean tumor size was $11.6 \pm 5.6 \mathrm{~cm}$. Six (12.2\%) of the patients were stage IIIB, 28 (57.1\%) were stage IIIC and 15 (30.6\%) were stage IVB. All patients underwent hysterectomy, bilateral salpingoophorectomy, pelvic paraaortic lymph node dissection and omentectomy as primary surgery. Hypertermic intraperitoneal chemotherapy (HIPEC) was administered to $55.1 \%$ of the patients and $10.2 \%$ of the patients underwent appendectomy. Peritonectomy procedure was added to $24.4 \%$ of the patients. Before surgery, $10.2 \%$ of patients received neoadjuvant $\mathrm{CT}$. Adjuvant paclitaxel + carboplatin was administered to $98.0 \%$ of the patients and $14.3 \%$ of the patients received neoadjuvant CT. CT could not be given to one patient due to her medical status. $10.2 \%$ of the patients who underwent resection + end-to-end anastomosis also had ileum resection. The mean number of pelvic lymph nodes (PLN) removed was $21.1 \pm 11.4$ and the number of paraaortic lymph nodes (PaLN) was $19.0 \pm 10.2$. PLN involve-

\begin{tabular}{|l|c|c|}
\hline Table 1. Clinical features of patients & \multicolumn{2}{|l|}{} \\
\hline & $\mathbf{n}$ & $\begin{array}{c}\text { Percentage } \\
\text { (\%) }\end{array}$ \\
\hline Intestinal surgery during primary operation & & \\
\hline Tumor excision & 7 & 14.3 \\
\hline Resection + ostomy & 34 & 69.4 \\
\hline Resection + end to end anastomosis & 8 & 16.3 \\
\hline Parts of intestinal surgery & & \\
\hline Sigmoid colon, rectum & 40 & 81.6 \\
\hline Right colon & 4 & 8.2 \\
\hline Transvers colon & 3 & 6.1 \\
\hline lleum & 2 & 4.1 \\
\hline Operation efficiency & & \\
\hline Suboptimal & 15 & 30.6 \\
\hline Optimal & 34 & 69.4 \\
\hline Histological type & & \\
\hline Epithelial cell & 46 & 93.9 \\
\hline Germ cell & 2 & 4.1 \\
\hline Sex-cord stromal tumor & 1 & 2.0 \\
\hline Intestinal layer involvement & & \\
\hline Serosa & 38 & 77.6 \\
\hline Muscularis & 7 & 14.3 \\
\hline Mucosa & 8.2 \\
\hline
\end{tabular}


ment was seen in $36.7 \%$ of the patients with PaLN in $36.7 \%$ of the patients.

Survival time and recurrence rates according to the type of intestinal surgery, the intestinal layer invaded by the tumor, and the efficiency of the surgery are shown in Table 2. Survival curves according to intestinal surgery type, the intestinal layer invaded by the tumor and the efficiency of surgery are shown in Figures 1, 2 and 3, respectively.

Risk factors affecting the OS interval were investigated according to Cox' regression analysis. Optimality of the primary CS ( $p=0.008$ and $H R=5.202)$ and stage $(p=0.016$ and $\mathrm{HR}=6.083$ ) were found to be statistically significant factors (Table 3).

Bladder laceration was seen in $6.7 \%$ of patients who underwent suboptimal surgery, and in $2.9 \%$ in patients whose surgery was optimal $(p=0.544)$. Ileus was observed in $13.3 \%$ of the patients who underwent suboptimal sur- gery. In the optimal surgical group, anastomotic leakage developed in 5.9\%, wound infection in 5.9\% and cardiac arrest in $2.9 \%$ of the patients. There were no complications in the tumor excision group. However, in patients undergoing resection + ostomy, ileus, anastomotic leakage, wound infection and cardiac arrest were seen at rates of 2.9\%, 2.9\%, $2.9 \%$ and $2.9 \%$, respectively. In patients who underwent resection + end-to-end anastomosis, ileus, anastomosis leakage and wound side infection occurred at $12.5 \%, 12.5 \%$ and $12.5 \%$, respectively.

$42.8 \%$ of patients whose tumor was excised during their primary CS needed a repeat operation with a resection+ostomy procedure and $14.2 \%$ had a repeat tumor excision procedure. $25 \%$ of the patients who underwent resection+end-to-end anastomosis, had a repeat resection+ostomy procedure, while $8.8 \%$ of patients who had a resection+ostomy underwent a repetition of resection+ostomy.

\begin{tabular}{|c|c|c|c|}
\hline & DFS; means (month) \pm SD & OS; means (month) \pm SD & Recurrence; n (\%) \\
\hline $\begin{array}{l}\text { Intestinal operation } \\
\text { Tumor excision } \\
\text { Resection + ostomy } \\
\text { Resection + end-to-end anastomosis }\end{array}$ & $\begin{array}{l}(p=0.072) \\
13.3 \pm 4.3 \\
18.3 \pm 2.2 \\
25.5 \pm 5.3\end{array}$ & $\begin{array}{l}(p=0.333) \\
20.8 \pm 5.1 \\
38.6 \pm 6.6 \\
34.0 \pm 3.5\end{array}$ & $\begin{array}{c}(p=0.064) \\
6(85.7) \\
13(38.2) \\
3(37.5)\end{array}$ \\
\hline $\begin{array}{l}\text { Intestinal layer involvement } \\
\text { Serosa } \\
\text { Muscularis } \\
\text { Mucosa }\end{array}$ & $\begin{array}{r}(p=0.131) \\
20.1 \pm 2.0 \\
16.2 \pm 2.6 \\
5.2 \pm 0.8\end{array}$ & $\begin{array}{r}(p=0.221) \\
39.3 \pm 6.4 \\
19.3 \pm 2.5 \\
7.0 \pm 1.2\end{array}$ & $\begin{array}{l}(p=0.973) \\
17(44.7) \\
3(42.9) \\
2(50.0)\end{array}$ \\
\hline $\begin{array}{l}\text { Efficiency of surgery } \\
\text { Non-optimal } \\
\text { Optimal }\end{array}$ & $\begin{array}{l}(p=0.004) \\
12.1 \pm 3.3 \\
21.9 \pm 2.1\end{array}$ & $\begin{array}{l}(\mathbf{p}=\mathbf{0 . 0 0 2}) \\
20.9 \pm 7.1 \\
30.3 \pm 2.6\end{array}$ & $\begin{array}{c}(p=0.158) \\
9(60.0) \\
13(38.2)\end{array}$ \\
\hline
\end{tabular}

DFS - disease free survival; OS — overall survival; SD — standard deviation
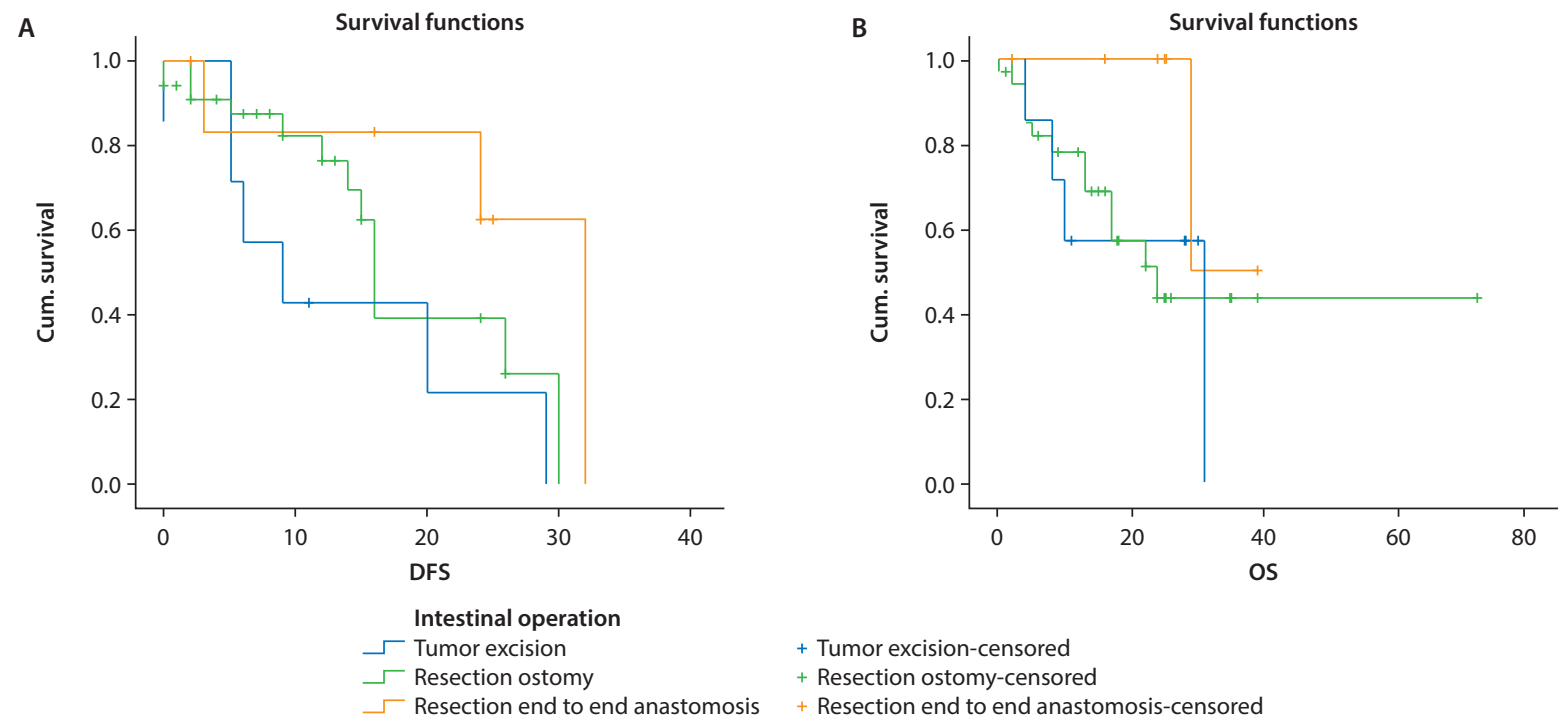

\footnotetext{
+ Tumor excision-censored

- Resection ostomy-censored

+ Resection end to end anastomosis-censored
}

Figure 1A. Disease free survival $(p=0.072)$ and B. Overall survival curves $(p=0.333)$ according to the type of intestinal surgery performed during primary cytoreductive surgery 
A

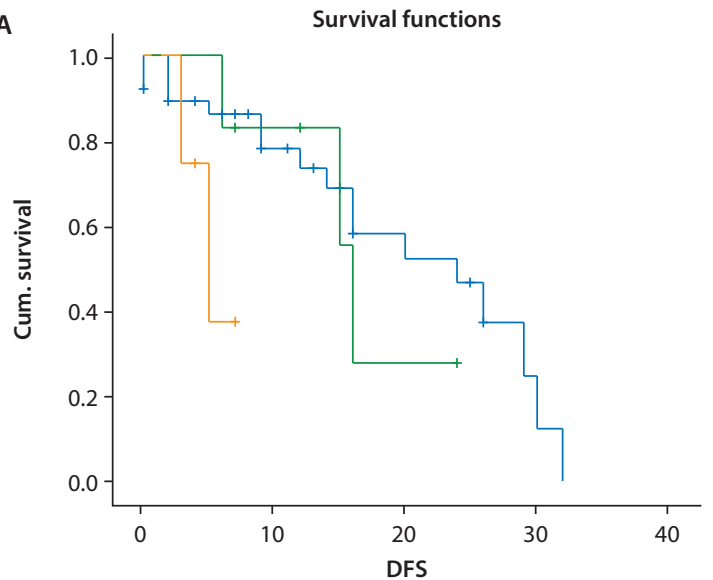

Intestinal layer

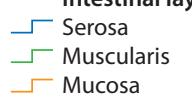

B

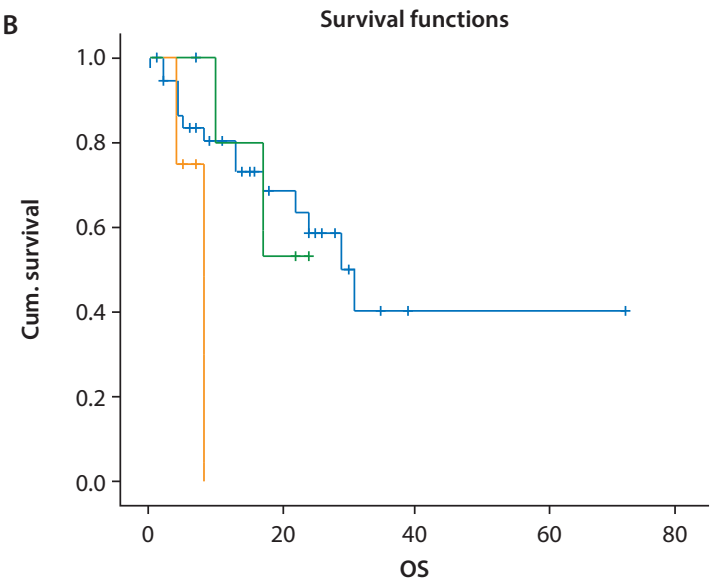

- Serosa-censored

+ Muscularis-censored

Mucosa-censored

Figure 2A. Disease free survival $(p=0.131)$ and B. Overall survival $(p=0.221)$ curves according to tumor expansion over intestinal layers

A

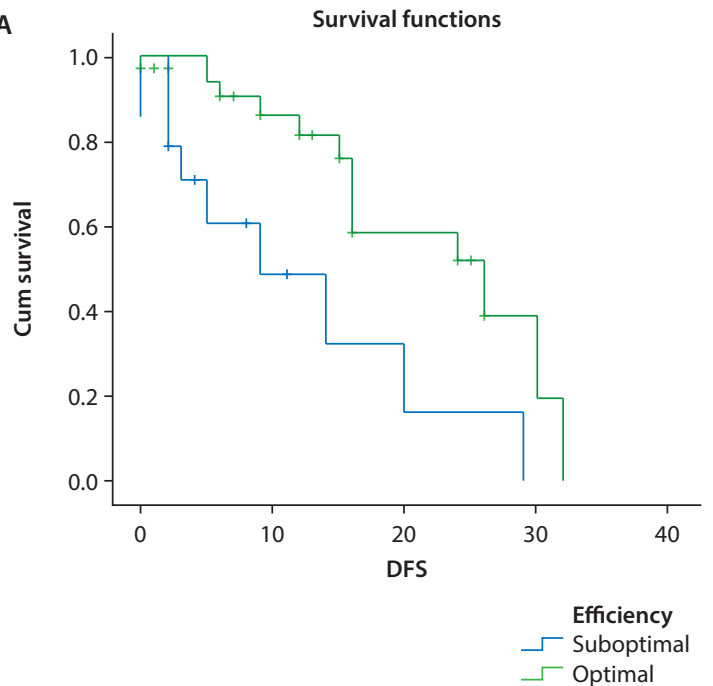

B

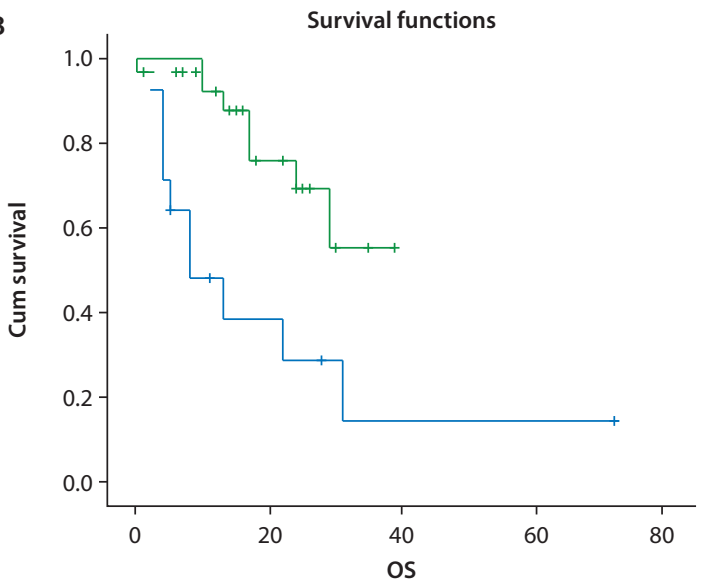

+ Suboptimal-censored

+ Optimal-censored

Figure 3A. Disease free survival $(p=0.004)$ and B. Overall survival $(p=0.002)$ curves according to the efficiency of primary cytoreductive surgery

\section{DISCUSSION}

Forty-nine patients who underwent surgery for stage IIIB-IVB OC with intestinal involvement were investigated in this retrospective study. The effects of the type of surgery and the success of optimal cytoreduction on the recurrence of DFS and OS were evaluated. A surgical approach to the gastrointestinal system is frequently required in $\mathrm{OC}$ surgery. Studies report the success of CS in stage II, III, and IV cancer cases as between $41 \%$ and $85 \%[8,9]$. In our study, the optimal cytoreduction rate in IIIB-IVB cases performed by a duo of general surgeon and gyneco-oncologist was found to be $74.2 \%$.

In a study that evaluated intestinal resection and end-to-end anastomosis performed due to the spread of
OC through the intestinal system, $48 \%$ of the patients demonstrated DFS and median survival time was 32 months [10]. The report shows that $5 \%$ of patients needed a secondary colostomy, while $25 \%$ of patients underwent anterior resection and reanastomosis [10]. When looking at different types of surgery, we found no differences regarding DFS $(p=0.072)$, OS $(p=0.333)$ and recurrence rates $(p=0.064)$ between the tumor excision, resection + ostomy and resection + end-to-end anastomosis groups. However, there were differences in terms of complications. While there were no complications in the tumor excision group, 2.9\% ileus and $2.9 \%$ anastomotic leakage rates were reported in patients having resection + ostomy; and $12.5 \%$ ileus and $12.5 \%$ anastomosis leakage were reported in patients who underwent 
Table 3. The relationship between OS interval and risk factors according to Cox' regression analysis

\begin{tabular}{|l|l|l|l|}
\hline & $\mathbf{P}$ & Hazard ratio & $\mathbf{9 5 \%} \mathbf{C I}$ \\
\hline Age & 0.922 & 1.0 & $0.9-1.0$ \\
\hline Type of Intestinal procedure & 0.805 & 1.2 & $0.2-6.0$ \\
\hline Additional ileum involvement & 0.446 & 0.2 & $0.1-8.1$ \\
\hline Intestinal site & 0.416 & 1.3 & $0.6-2.8$ \\
\hline HIPEC & 0.395 & 3.6 & $0.1-72.3$ \\
\hline Efficiency of primary CS & $\mathbf{0 . 0 0 8}$ & $\mathbf{5 . 2}$ & $1.5-17.5$ \\
\hline Histological type & 0.063 & 1.7 & $0.9-2.9$ \\
\hline Intestinal layer & 0.096 & $0.1-1.2$ \\
\hline Tumor size & 0.569 & 0.3 & $0.9-1.2$ \\
\hline Stage & $\mathbf{0 . 0 1 6}$ & 1.0 & $1.4-26.3$ \\
\hline Hemoglobin & 0.901 & $\mathbf{6 . 0}$ & $0.5-2.1$ \\
\hline Ca125 & 0.793 & 1.0 & $0.9-1.0$ \\
\hline Neo-adjuvant chemotherapy & 0.985 & 1.0 & $0.0-0.1$ \\
\hline
\end{tabular}

$\mathrm{Cl}$ - onfidence intervals; HIPEC — hyperthermic intraperitoneal chemotherapy; CS — cytoreductive surgery

Resection + end-to-end anastomosis. Of those patients who had a tumor excision during their primary $\mathrm{CS}, 42.8 \%$ underwent a repetition using the resection+ostomy procedure; and $14.2 \%$ had a repeated tumor excision procedure. $25 \%$ of the patients who underwent resection+end-to-end anastomosis had a repeated resection+ostomy procedure, while $8.8 \%$ of the patients having resection+ostomy underwent a repeated resection+ostomy.

Reports have shown that patients with a rectal invasion through the serosa and above the serosa layer have longer DFS intervals than patients with muscularis and mucosa layer invasions; however, there is no significant difference with regard to the OS interval [10]. We calculated DFS intervals $(p=0.131)$ according to tumor presence in the serosa, muscularis or mucosa layers as 20.1-16.2-5.2 months, respectively; and OS intervals $(p=0.221)$ as $39.3-19.3-7.0$ months, respectively.

In a metaanalysis of 6,685 patients, it was seen that an increase of $10 \%$ in CS enhanced the mean survival rate by $5.5 \%$ [11]. The mean OS interval for patients with a total resection was 72 months and for those with a residual tumor 42 months [8]. $4.7 \%$ of patients who underwent optimal cytoreduction and $60 \%$ of patients with a suboptimal cytoreduction had pelvic recurrence [9]. Patients without a residual tumor had longer DFS and OS intervals than patients with a residual tumor [12]. Another study showed no significant difference between the two groups (total rectosigmoid resection group and partial rectosigmoid resection group) regarding 5-years OS rates which were 48\% and $52 \%$, respectively [13]. Patients undergoing optimal cytoreduction had significantly longer OS and DFS intervals according to our study. However, there was no significant difference between the two groups regarding recurrence rates. Bladder laceration was seen in $6.7 \%$ of patients who underwent suboptimal surgery and in $2.9 \%$ of patients who underwent optimal surgery $(p=0.544)$. lleus was observed in $13.3 \%$ of patients who underwent suboptimal surgery. $5.9 \%$ anastomotic leakage, 5.9\% wound infection and $2.9 \%$ cardiac arrest developed in the optimal surgery group.

The study had several limitations: first, its retrospective nature; secondly, its small sample size. Despite these limitations, our hospital is a tertiary center for gynecologic oncology in the Aegean region, and many patients visit our clinic from distant locations. Therefore, the similarity of demographic characteristics in the study population, availability of good follow-up data, and performance of surgeries by the same surgical team increased the validity of results and mitigated weaknesses.

\section{CONCLUSIONS}

Achieving optimal cytoreduction is the most important aim for the general surgeon carrying out an intestinal resection procedure. Although resection procedures are superior in providing the desired optimal results when compared to excision surgery, their higher complication rates and subsequent lower quality of life must be taken into consideration when choosing either resection or excision methods; surgical intervention should always be kept to the minimum possible. However, further studies are needed to clarify the exact role to be played by the general surgeon in this situation.

\section{Conflict of interest}

The authors have no commercial associations or sources of support in the forms of grants, equipment, drugs, or all of these that might pose a conflict of interest. All authors 
have made substantive contributions to the study, and all authors endorse the data and conclusions.

\section{REFERENCES}

1. Landis SH, Murray T, Bolden S, et al. Cancer statistics, 1999. CA Cancer J Clin. 1999; 49(1): 8-31, doi: 10.3322/canjclin.49.1.8.

2. Vidal F, Guerby P, Luyckx M, et al. Are Early Relapses in Advanced-Stage Ovarian Cancer Doomed to a Poor Prognosis? PLoS One. 2016; 11(1): e0147787, doi: 10.1371/journal.pone.0147787, indexed in Pubmed: 26820579.

3. Minig L, Zorrero C, Iserte PP, et al. Selecting the best strategy of treatment in newly diagnosed advanced-stage ovarian cancer patients. World J Methodol. 2015; 5(4): 196-202, doi: 10.5662/wjm.v5.i4.196, indexed in Pubmed: 26713279.

4. Pohl R, Dallenbach-Hellweg G, Plügge T, et al. Prognostic parameters in patients with advanced ovarian malignant tumors. Eur J Gynaecol Oncol. 1984; 5(3): 160-169, indexed in Pubmed: 6329760.

5. Kato K, Nishikimi K, Tate S, et al. Histopathologic tumor spreading in primary ovarian cancer with modified posterior exenteration. World J Surg Oncol. 2015; 13: 230, doi: 10.1186/s12957-015-0647-x, indexed in Pubmed: 26228239.

6. Look M, Chang D, Sugarbaker PH. Long-term results of cytoreductive surgery for advanced and recurrent epithelial ovarian cancers and papillary serous carcinoma of the peritoneum. Int J Gynecol Cancer. 2004; 14(1): 35-41, doi: 10.1111/j.1048-891x.2004.14008.x.
7. Randall TC, Rubin SC. Cytoreductive surgery for ovarian cancer. Surg Clin North Am. 2001; 81(4): 871-883, doi: 10.1016/s0039-6109(05)70171-7, indexed in Pubmed: 11551131

8. Peiretti M, Bristow RE, Zapardiel I, et al. Rectosigmoid resection at the time of primary cytoreduction for advanced ovarian cancer. A multi-center analysis of surgical and oncological outcomes. Gynecol Oncol. 2012; 126(2): 220-223, doi: 10.1016/j.ygyno.2012.04.030, indexed in Pubmed: 22555105.

9. Hertel $\mathrm{H}$, Diebolder $\mathrm{H}$, Herrmann J, et al. Is the decision for colorectal resection justified by histopathologic findings: a prospective study of 100 patients with advanced ovarian cancer. Gynecol Oncol. 2001; 83(3): 481-484, doi: 10.1006/gyno.2001.6338, indexed in Pubmed: 11733959

10. Sonnendecker EW, Beale PG. Rectosigmoid resection without colostomy during primary cytoreductive surgery for ovarian carcinoma. Int Surg. 1989; 74(1): 10-12, indexed in Pubmed: 2707991.

11. Bristow RE, Tomacruz RS, Armstrong DK, et al. Survival effect of maximal cytoreductive surgery for advanced ovarian carcinoma during the platinum era: a meta-analysis. J Clin Oncol. 2002; 20(5): 1248-1259, doi: 10.1200/JCO.2002.20.5.1248, indexed in Pubmed: 11870167.

12. Park JY, Seo SS, Kang S, et al. The benefits of low anterior en bloc resection as part of cytoreductive surgery for advanced primary and recurrent epithelial ovarian cancer patients outweigh morbidity concerns. Gynecol Oncol. 2006; 103(3): 977-984, doi: 10.1016/j.ygyno.2006.06.004, indexed in Pubmed: 16837030

13. Plotti F, Montera R, Aloisi A, et al. Total rectosigmoidectomy versus partial rectal resection in primary debulking surgery for advanced ovarian cancer. Eur J Surg Oncol. 2016; 42(3): 383-390, doi: 10.1016/j. ejso.2015.12.001, indexed in Pubmed: 26725211. 